\title{
One-Step Multiplex PCR for Simultaneous Detection and Identification of Eight Medically Important Candida Species
}

\author{
Akira Fukatsu ${ }^{*}$, Osamu Tsuzukibashi', Hidenori Suzuki², Katsuhiro Asaka², Yoshinori Ono², \\ Mana Fuchigami' ${ }^{1}$, Taira Kobayashi ${ }^{3}$, Satoshi Uchibori ${ }^{3}$, Yuji Takahashi ${ }^{3}$, Chiaki Komine', \\ Yoshimi Konishi' ${ }^{1}$, Yuki Ogura', Hiroko Omori' ${ }^{2}$, Masanobu Wakami ${ }^{4}$, Hiroshi Murakami ${ }^{3}$, \\ Masahiko Fukumoto ${ }^{1}$
}

\begin{abstract}
${ }^{1}$ Division of Laboratory Medicine for Dentistry, Department of Oral Health Science, Nihon University School of Dentistry at Matsudo, Chiba, Japan

${ }^{2}$ Laboratory Medicine for Dentistry, Nihon University Graduate School of Dentistry at Matsudo, Chiba, Japan

${ }^{3}$ Department of Fixed Prosthodontics and Oral Implantology, Nihon University School of Dentistry at Matsudo, Chiba, Japan ${ }^{4}$ Division of Oral Function and Rehabilitation, Department of Oral Health Science, Nihon University School of Dentistry at Matsudo, Chiba, Japan

Email: *fukatsu.akira@nihon-u.ac.jp
\end{abstract}

How to cite this paper: Fukatsu, A., Tsuzukibashi, O., Suzuki, H., Asaka, K., Ono, Y., Fuchigami, M., Kobayashi, T., Uchibori, S., Takahashi, Y., Komine, C., Konishi, Y., Ogura, Y., Omori, H., Wakami, M., Murakami, H. and Fukumoto, M. (2021) OneStep Multiplex PCR for Simultaneous Detection and Identification of Eight Medically Important Candida Species. Open Journal of Stomatology, 11, 14-24.

https://doi.org/10.4236/ojst.2021.111002

Received: December 10, 2020

Accepted: January 23, 2021

Published: January 26, 2021

Copyright $\odot 2021$ by author(s) and Scientific Research Publishing Inc. This work is licensed under the Creative Commons Attribution International License (CC BY 4.0)

http://creativecommons.org/licenses/by/4.0/

\begin{abstract}
Recently, the incidence of Candida infections has substantially increased. Conventional identification methods for Candida species are technically difficult to conduct and cannot accurately distinguish each species. The purpose of the present study was to design primers to identify and detect simultaneously eight medically important Candida species using one-step multiplex PCR. PCR primers were designed based on partial sequences of intergenic spacer (IGS) and internal transcribed spacer (ITS) genes of eight medically important Candida species. These primers were able to distinguish each Candida species and did not display cross-reactivity with representative Candida species other than the eight Candida species. Moreover, our developed one-step multiplex PCR method is accurate, specific, cost-effective, time-saving, and worked without requiring DNA extraction.
\end{abstract}

Keywords

Candida, Candida albicans, One-Step Multiplex PCR, PCR Method

\section{Introduction}

The genus Candida belongs to the kingdom Fungi, class Deuteromycetes, and comprises 150 - 200 species. Recently, distinct shifts in the distribution of Can- 
dida species isolated from nosocomial infections have been reported. Although Candida albicans remains the most frequent cause of candidemia and hematogenously disseminated candidiasis, an increasing number of hospital-acquired infections caused by other Candida species, so-called non-albicans Candida species, is being observed [1] [2] [3] [4] [5]. Candidemia is often associated with human immunodeficiency virus (HIV) or advanced medical and surgical interventions that compromise patient immunity, e.g., bone marrow or solid organ transplant, aggressive chemotherapy, and broad application of antifungal agents [6]. In fact, nosocomial fungal bloodstream infections are an increasingly significant cause of morbidity, with an estimated mortality of $25 \%-38 \%$ [7]. C. albicans is the most common and clinically relevant pathogen of the genus. However, there has been a significant upward trend in the emergence of non-albicans Candida species, especially Candida glabrata, Candida parapsilosis, and Candida tropicalis [8] [9] [10]. In addition, because several non- $C$. albicans Candida species are frequently resistant to common antifungal agents, accurate identification methods are essential for the establishment of appropriate antifungal therapy [11].

The basis for laboratory detection of bloodstream fungal infections, including candidemia, remains direct examination and conventional blood culture. However, these methods are of limited clinical value because there are negative outcomes in as high as $50 \%$ of autopsy-confirmed cases of candidemia. In addition, cultures may only become positive late in the infection [12]. Furthermore, most phenotypic methods of identification used in clinical laboratories are often timeconsuming and may lead to inconclusive results. For example, phenotypic tests such as VITEK and API ID32C systems need several days before biochemical reactions can be interpreted [13]. In contrast, molecular approaches have the potential to detect candidemia swiftly with increased sensitivity and specificity. Buchman et al. demonstrated first that detection of $C$. albicans in clinical specimens was possible by PCR amplification of the lanosterol-alpha-demethylasegene [14]. Other PCR-based techniques have been developed using amplification of target DNA, providing alternative strategies for the diagnosis and identification of fungal pathogens [15] [16] [17] [18]. Fungal ribosomal genes are common DNA targets in PCR-based procedures for the identification of fungi at the species level. The highly variable sequences of internal transcribed spacer (ITS) regions ITS1 and ITS2 flanked by the relatively conserved coding regions of $18 \mathrm{~S}, 5.8 \mathrm{~S}$, and $28 \mathrm{~S}$ nuclear rRNA genes have been used in various PCR-based approaches for the identification of medically important yeasts [19] [20] [21]. Although molecular techniques such as the conventional PCR method are highly sensitive and specific, they are also expensive, laborious, and a little time-consuming. Thus, a simple and more reliable assay for identifying Candida species is desired.

The purpose of the present study was to develop a one-step multiplex PCR system for identifying and detecting simultaneouslyeight medically important Candida species, i.e., C. albicans, C. glabrata, C. tropicalis, C. parapsilosis, Candida dubliniensis, Candida guilliermondii (currently Pichia guilliermondii), Can- 
dida krusei (currently Issatchenkia orientalis), and Candida lusitaniae (currently Clavispora lusitaniae).

\section{Materials and Methods}

\subsection{Fungal Strains and Culture Conditions}

All microorganisms were obtained from Japan Collection of Microorganisms (JCM; Japan). The following fungal strains were used in the present study: $C$. albicans JCM 1537, C. glabrata JCM 1539, C. tropicalis JCM 1541, C. parapsilosis JCM 1612, C. dubliniensis IFM 54605, C. guilliermondii JCM 1539, C. krusei JCM 1609, C. lusitaniae JCM 1814, Candida kruisii JCM 1779, Candida orthopsilosis, Candida kefyr JCM 9556, Candida aaseri JCM 1689, Candida inconspicua JCM 9555, Candida melibiosica JCM 9558, and Candida norvegica JCM 8897. These strains were maintained by cultivation on Bacto ${ }^{\mathrm{TM}}$ Brain Heart Infusion (BHI; Becton Dickinson and Co., Sparks, MD, USA) and 1.5\% agar (BHI agar). The organisms were cultured overnight at $30^{\circ} \mathrm{C}$ under aerobic condition.

\subsection{Design of Species-Specific Primers for Eight Species}

Design of species-specific primers for eight species was performed as described previously [22]. Briefly, the intergenic spacer (IGS) gene sequences of C. albicans (accession no. FN554375), C. glabrata (FN554379), C. tropicalis (FN554382), C. parapsilosis (FN554241), C. dubliniensis (FN554377), and C. guilliermondii (AM992960) and internal transcribed spacer (ITS) gene sequences of $C$. albicans (accession No. AF217609), C. glabrata (KJ546151), C. tropicalis (KY495750), C. parapsilosis (KY685084), C. dubliniensis (KX231794), C. guilliermondii (AF022717), C. lusitaniae (AF009215), and C. krusei (AF246989) were obtained from the DNA Data Bank of Japan (DDBJ; https://www.ddbj.nig.ac.jp/services.html, Mishima, Japan), and a multiple sequence alignment analysis was performed using the CLUSTAL W program; i.e., IGS gene sequences of six Candida species and ITS gene sequences of eight Candida species were aligned and analyzed. Homology among the primers selected for each Candida species and their respective IGS and ITS gene sequences was confirmed by a BLAST search.

\subsection{Development of a One-Step Multiplex PCR Method Using Designed Primers}

Fungal cells were cultured in BHI supplemented with $0.5 \%$ yeast extract for $24 \mathrm{~h}$, and 1-ml samples were then collected in microcentrifuge tubes and resuspended at a density of $1.0 \mathrm{McF}$ arland standard [approximately $10^{7}$ colony-forming units $(\mathrm{CFU}) / \mathrm{ml}$ in $1 \mathrm{ml}$ of sterile distilled water. A total of $3.6 \mu \mathrm{l}$ of the suspension was then used as the PCR template. The detection limit of PCR was assessed by serially diluting known numbers of fungal cells in sterile distilled water and then subjecting each suspension to PCR. The multiplex PCR mixture contained 0.2 $\mu \mathrm{M}$ of each primer, $10 \mu \mathrm{l}$ of $2 \times$ MightyAmp Buffer Ver.3 (Takara Bio Inc., Shiga, Japan), $0.4 \mu$ lof MightyAmp DNA Polymerase (Takara), and $5 \mu \mathrm{l}$ of the template 
in a final volume of $20 \mu \mathrm{l}$. PCRs were performed in a DNA thermal cycler (Applied Biosystems 2720 Thermal Cycler; Applied Biosystems, Foster City, CA, USA). PCR conditions included an initial denaturation step at $98^{\circ} \mathrm{C}$ for $2 \mathrm{~min}$, followed by 30 cycles consisting of $98^{\circ} \mathrm{C}$ for $10 \mathrm{~s}$ and $68^{\circ} \mathrm{C}$ for $1 \mathrm{~min}$. PCR products were analyzed by $2.0 \%$ agarose gel electrophoresis and then visualized by electrophoresis in $1 \times$ Tris-borate-EDTA on a $2 \%$ agarose gel stained with ethidium bromide. A 100-bp DNA ladder (Takara Biomed, Shiga, Japan) was used as a molecular size marker. All experiments were performed in triplicate.

\section{Results}

\subsection{Primer Design}

Sixteen specific primers covering the upstream regions of IGS and ITS gene sequences of eight medically important Candida species were designed in the present study (Figure 1 and Figure 2). The specific forward primers were designated as CLF1 for C. lusitaniae, PKF1 for C. krusei, CGLF1 for C. glabrata, CTF1 for C. tropicalis, $\mathrm{CPF} 1$ for C. parapsilosis, $\mathrm{CDF} 1$ for $C$. dubliniensis, CGUF1 for C. guilliermondii, and CAF1 for C. albicans. The specific reverse primers were designated as CLF2 for C. lusitaniae, PKF2 for C. krusei, CGLF2 for C. glabrata, CTF2 for C. tropicalis, and CPF2 for C. parapsilosis, CDF2 for C. dubliniensis, CGUF2 for $C$. guilliermondii, and CAF2 for $C$. albicans. Amplicon sizes of $C$. lusitaniae, C. krusei, C. glabrata, C. tropicalis, and C. parapsilosis, C. dubliniensis, C. guilliermondii, and C. albicans were 125 bp, 230 bp, 331 bp, 424 bp, 507 bp, $684 \mathrm{bp}, 816 \mathrm{bp}$, and $1009 \mathrm{bp}$, respectively.

\subsection{Multiplex PCR}

\subsubsection{Detection Limit}

Our multiplex PCR method for identifying and detecting eight medically important Candida species, i.e., C. albicans, C. glabrata, C. tropicalis, C. parapsilosis, C. dubliniensis, C. guilliermondii, C. krusei, and C. lusitaniae, successfully amplified DNA fragments of the expected size for each species (Figure 3). The detection limit was assessed in the presence of titrated fungal cells, and the sensitivity of the PCR assay was 5 - $50 \mathrm{CFU}$ per PCR template $(5.0 \mu \mathrm{l})$ for the C. Iusitaniae-specific primer set with strain JCM 1814, the C. krusei-specific primer set with strain JCM 1609, the C. glabrata-specific primer set with strain JCM 1539, the C. tropicalis-specific primer set with strain JCM 1541, the C. parapsilosis-specific primer set with strain JCM 1612, the C. dubliniensis-specific primer set with strain IFM 54605, the $C$. guilliermondii-specific primer set with strain JCM 1539, and the C. albicans-specific primer set with strain JCM 1537 (Figure 4 and Figure 5).

\subsubsection{Assay of Representative Candida Species Other than the Eight Medically Important Species}

As representative Candida species other than the eight species targeted in this study, C. kruisii, C. orthopsilosis, C. kefyr, C. aaseri, C. inconspicua, C. melibiosica, 


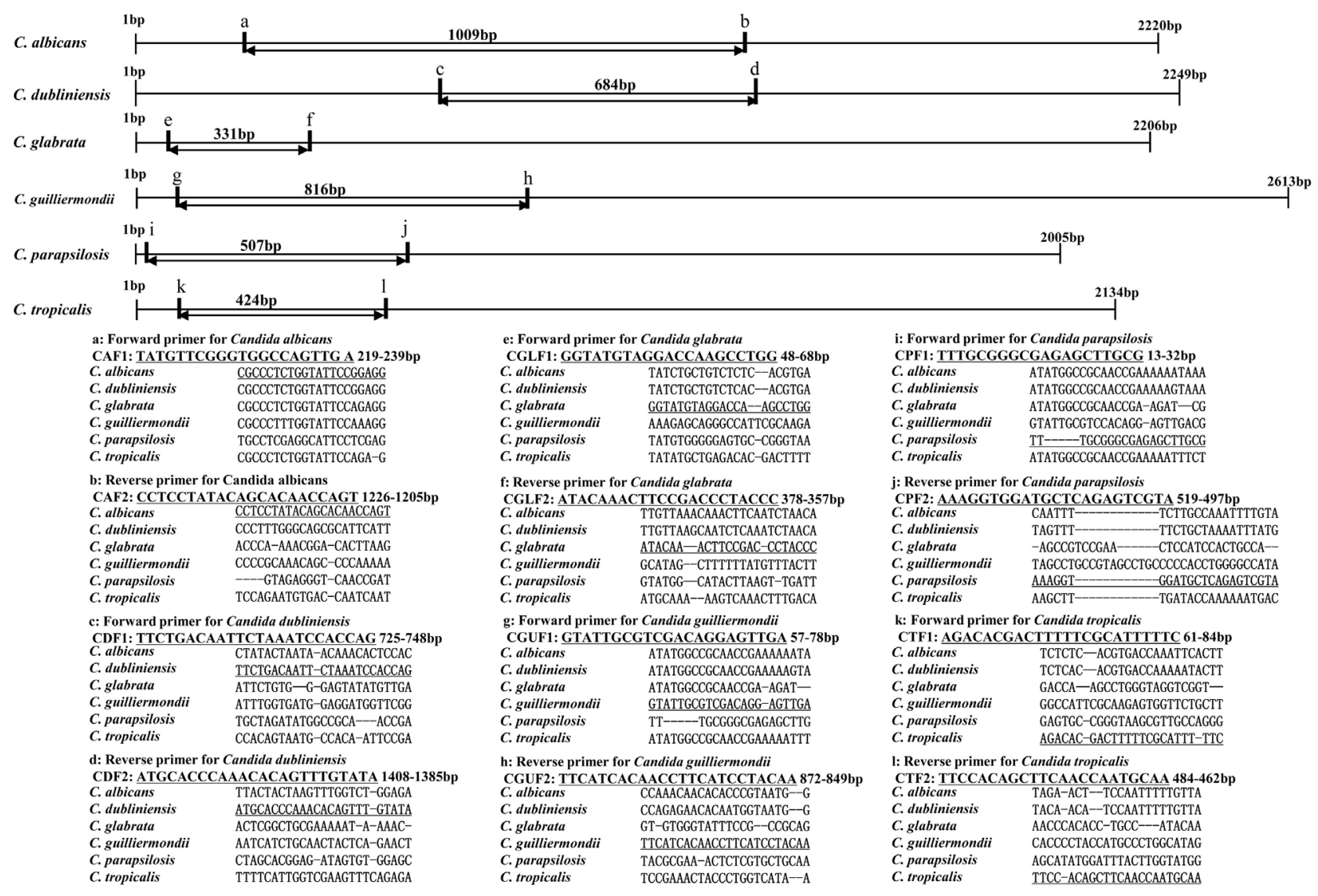

Figure 1. Locations and sequences of species-specific primers for IGS genes of C. albicans, C. dubliniensis, C. glabrata, C. guilliermondii, C. parapsilosis, and C. tropicalis. The nucleotide sequence of each primer is underlined.

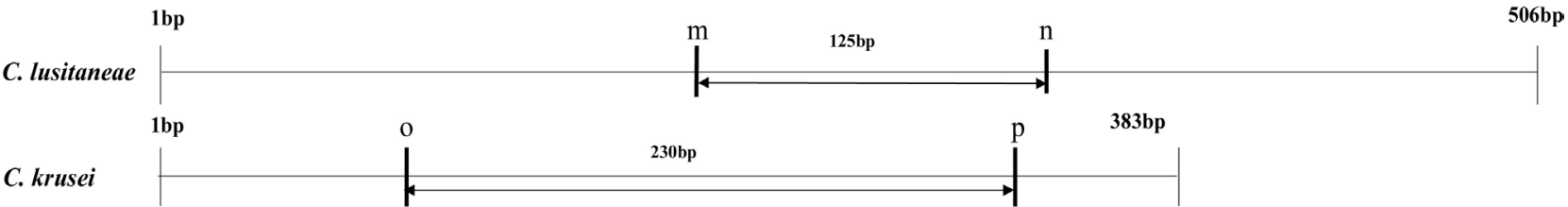

m: Forward primer for Candida lusitaneae

CLF1: TGCCTCGAGGCATTCCTCGAG 209-229bp

$\begin{array}{ll}\text { C. } \text { albicans } & \text { CGCCCTCTGGTATTCCGGAGG } \\ \text { C. } \text { dubliniensis } & \text { CGCCCTCTGGTATTCGGAGG } \\ \text { C. } \text { glabrata } & \text { CGCCCTCTGGTATTCGGGGG } \\ \text { C. } \text { guilliermondii } & \text { CGCCCTCTGGTATTCAGA-G } \\ \text { C. } \text { krusei } & \text { CGCCCCTCGGCATTCCGGGGG } \\ \text { C. lusitaneae } & \text { TGCCTCGAGGCATTCCTCGAG } \\ \text { C. parapsilosis } & \text { CGCCCTCTGGTATTCCAGAGG } \\ \text { C. } \text { tropicalis } & \text { CGCCCTTTGGTATTCCAAAGG }\end{array}$

\section{n: Reverse primer for Candida lusitaneae}

\section{CLF2: GAGGGCGAAATGTGGTGCTGTA 333-312bp}

C. albicans GAGGTCAA----A-GTTTGAAGATATACGTG-GTGGACGTT---ACCGCCGCAAGC

C. dubliniensis GAGGTC-A---A-GTTTGAAGAATAAAATG - GGCGACGCCAGAGACCGCCTT-AGC

C. glabrata AGTATTAACCCCCGCCGCTCGCGCAAACGAGCAGCAGATTAAT--_-_-AGA

C. guilliermondii CCCGCCAC-- -ACCATTCAACGAGTTGGATA-AACCTAAT-- -AC- —-ATT

c. krusei GAGGTCGA-_-GCTTTTTGTTGTCTCGCA- - ACACTCGCTCTCGGCCG-CCA

C. lusitaneae
C. parapsilosis

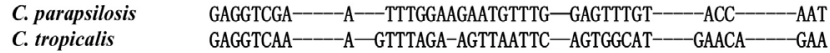

o: Forward primer for Candida krusei

CKF1: TGTGGAATATAGCATATAGTCGACA 108-132bp

C. albicans

C. dubliniensis

C. glabrata

C. krusei

C. Iusitaneae

C. parapsilosis

C. tropicalis
CCAA-TTTTTT-A-TCAACTT-GTCA

CCAA-ATTTTTT--A-TAAACTT-GTCA

CAAATATTTTTTTAAACTAATTCAGTCA

TGTGGAATATAG--CATATAGTC-GACA

TAAA-TTTTTTT--ATTCGAATT--TC-

CCAA-ATTTTTT--A-TTAATT--GTCA

CCAA-ACTTTTT--ACTTGATTCAGTCA

p: Reverse primer for Candida krusei

CKF2: CAACTCTGCGCACGCGCAAGAT 337-316bp

AAACCC-AG--CGGTTTGAGGG
AAACCCTAG--GGGTTTGAGGG
AAACACAAT--GTGTTTGAGAA
AAACCCGGG---GGTTTGAGAG
CAACTCTGCGCACGCGCAAGAT
TAACC-GG--GGGTTAGAGGG
AAACCCGAA---GGTTTGAGGG
AAACCCGAG---GGTTTGAGGG

Figure 2. Locations and sequences of species-specific primers for ITS genes of $C$. lusitaniae and $C$. krusei. The nucleotide sequence of each primer is underlined. 


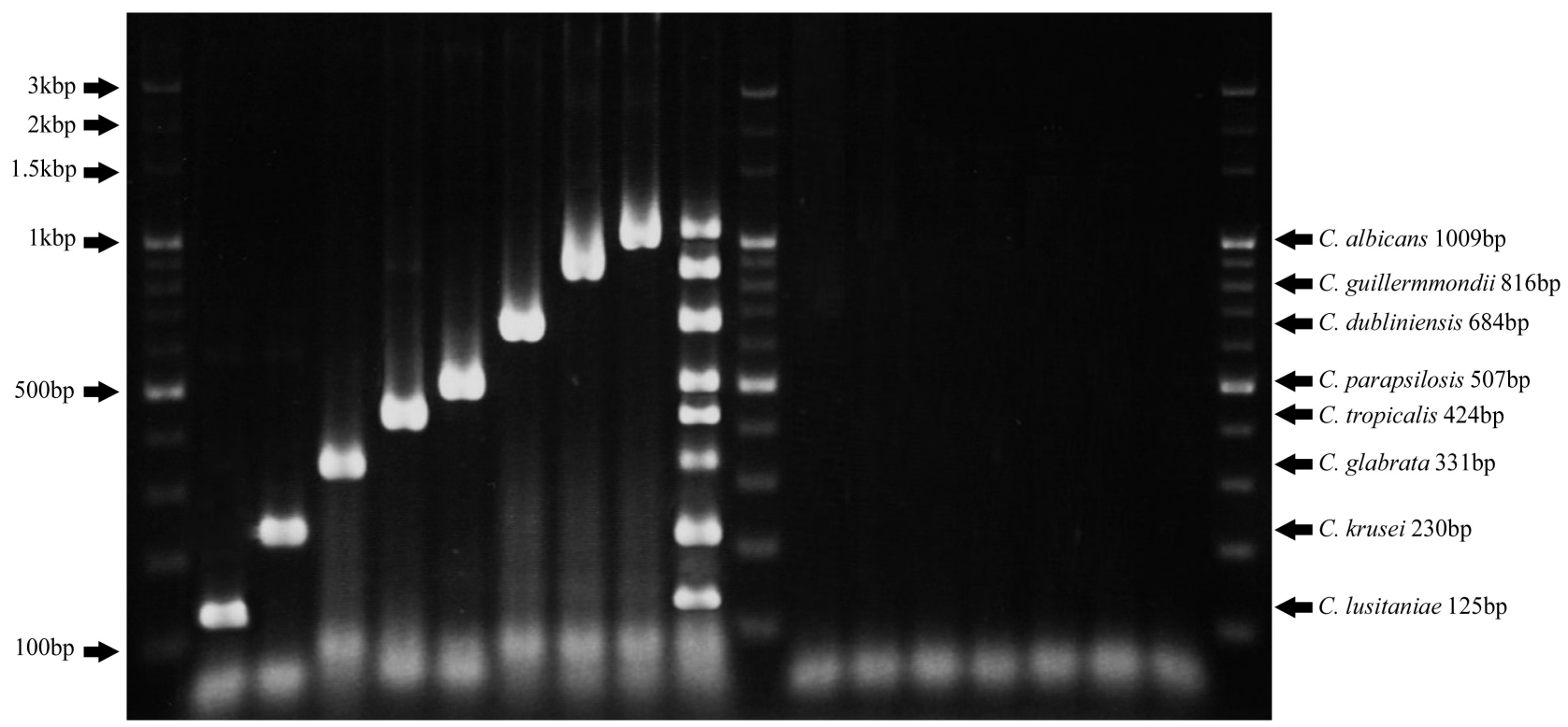

Figure 3. Multiplex PCR assay for detecting eight medically important Candida species. The primer mixture contained CLF1, CLF2, CPF1, CPF2, CKF1, CKF2, CGLF1, CGLF2, CDF1, CDF2, CTF1, CTF2, CAF1, CAF2, CGUF1, and CGUF2. Lanes: 1, Candida lusitaniae JCM 1814; 2, Candida krusei JCM1609; 3, Candida glabrata JCM3761; 4, Candida tropicalis JCM 1541; 5, Candida parapsilosis JCM 1612; 6, Candida dubliniensis IFM 54605; 7, Candida guilliermondii JCM 1539; 8, Candida albicans JCM 1537; 9, Mixture of C. lusitaniae, C. krusei, C. glabrata, C. tropicalis, C. parapsilosis, C. dubliniensis, C. guilliermondii, and C. albicans, 10, Candida kruisii JCM 1779; 11, Candida orthopsilosis JCM 1784; 12, Candida kefyr JCM 9556; 13, Candida aaseri JCM 1689; 14 , Candida inconspicua JCM 9555; 15, Candida melibiosica JCM 9558; and 16, Candida norvegica JCM 8897. M, molecular size marker (100-bp DNA ladder).

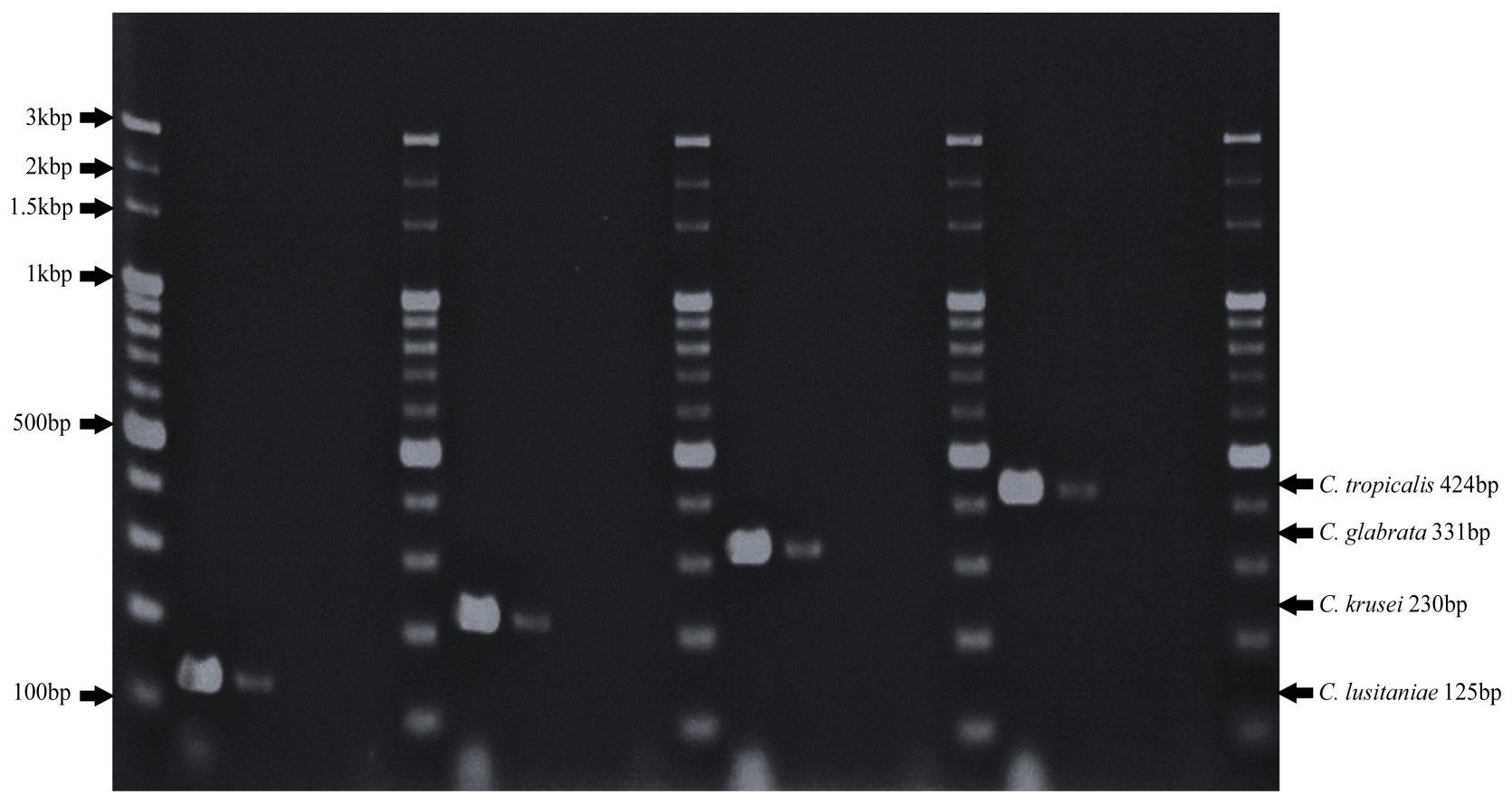

Figure 4. Detection limit of the multiplex PCR assay for detecting C. Iusitaniae, C. krusei, C. glabrata, and C. tropicalis. The primer mixture contained CLF1, CLF2, CPF1, CPF2, CKF1, CKF2, CGLF1, CGLF2, CDF1, CDF2, CTF1, CTF2, CAF1, CAF2, CGUF1, and CGUF2. Lanes 1 - 4, Candida lusitaniae JCM 1814; Lanes 5 - 8, Candida krusei JCM1609; Lanes 9 - 12 Candida glabrata JCM3761; Lanes 13 - 16 Candida tropicalis JCM 1541. The following numbers of cells were added: $5 \times 10^{2}$ (lanes $\left.1,5,9,13\right), 50$ (lanes 2, 6, 10,14), $5 \times 10$ (lanes 3, 7, 11, 15), and 0 (lanes 4, 8, 12, 16). M, molecular size marker (100-bp DNA ladder). 


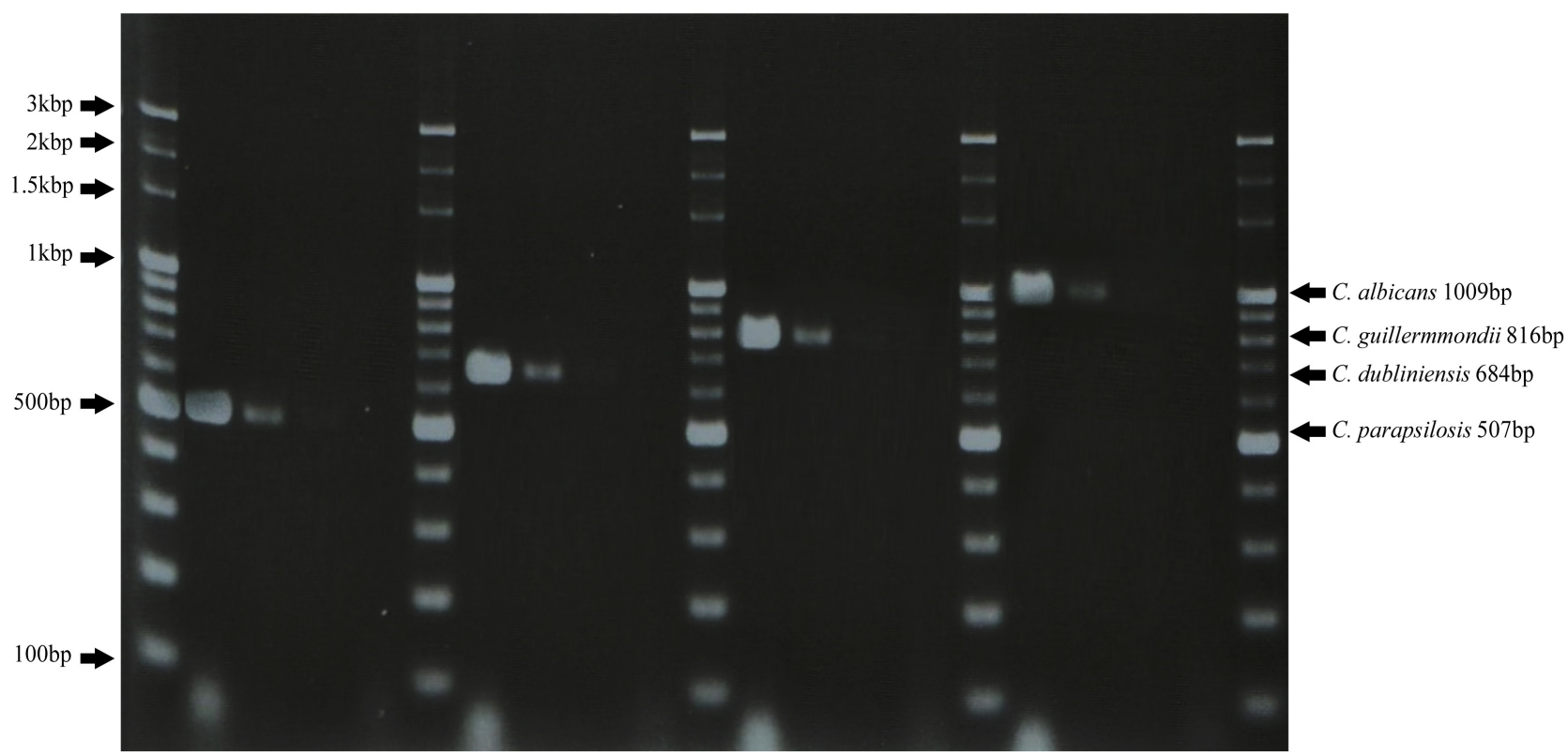

Figure 5. Detection limit of the multiplex PCR assay for detecting Candida parapsilosis, Candida dubliniensis, Candida guilliermondii, and Candida albicans. The primer mixture contained CLF1, CLF2, CPF1, CPF2, CKF1, CKF2, CGLF1, CGLF2, CDF1, CDF2, CTF1, CTF2, CAF1, CAF2, CGUF1, and CGUF2. Lanes 1 - 4, Candida parapsilosis JCM 1612; Lanes 5 - 8, Candida dubliniensis IFM 54605; Lanes 9 - 12 Candida dubliniensis IFM 54605; Lanes 13 - 16, Candida albicans JCM 1537. The following numbers of cells were added: $5 \times 10^{2}$ (lanes 1, 5, 9, 13), $5 \times 10$ (lanes 2, 6, 10, 14), $5 \times 1$ (lanes 3, 7, 11, 15), and 0 (lanes 4, 8, 12, 16). M, molecular size marker (100-bp DNA ladder).

and $C$. norvegica were evaluated by PCR using the designed primer sets. However, no amplicons were produced from any of the representative Candida species other than the eight medically important species (Figure 3 ).

\section{Discussion}

The incidence of Candida infections has recently increased due to the widespread use of broad-spectrum antibiotics and growing numbers of HIV-infected and immunocompromised individuals [23] [24] [25]. Despite the predominance of $C$. albicans, non-albicans Candida species such as C. glabrata, C. tropicalis, C. guilliermondii, C. dubliniensis, C. parapsilosis, C. krusei, and C. lusitaniae are emerging as both colonizers and pathogens that can cause systemic infections [26] [27] [28] [29]. Furthermore, some of these species are naturally more resistant to antifungal agents. C. glabrata and C. krusei are innately more resistant to the commonly used antifungal agent fluconazole [30]. Moreover, the genome of $C$. $d u b$ liniensis, which is mainly sensitive to fluconazole, encodes for multidrug transporters that rapidly mediate fluconazole resistance during clinical therapy [31].

Thus, rapid and accurate identification of disease-causing Candida species is crucial for clinical treatment of local or systemic candidiasis. Premature diagnosis of invasive fungal infections is problematic because most clinical signs and symptoms are non-specific, and cultures are often negative or become positive too late for the initiation of effective antifungal therapy. Therefore, several studies have been developed for improvement of new technologies for the diagnosis 
of invasive candidiasis. Conventional methodology has long been used as standard identification procedures for Candida species. However, these methods are laborious, time-consuming, and not reliable in identifying the broad spectrum of Candida species and usually require additional tests. Therefore, several commercial systems have been developed to enable rapid yeast identification within 2 72 hours. Although these systems have been extensively used for Candida identification, their application is limited, and some species cannot be identified and differentiated. Recently, molecular approaches, such as PCR-based methods, have been used to complement conventional methods and provide more accurate results in less time ( 2 - 3 hours). Given the high accuracy and speed with which molecular typing techniques can be carried out and rapid advances in technology, most of these methods may improve routine clinical laboratory identification of Candida species. However, further studies are needed for the standardization of such technical procedures.

To develop a PCR-based technique more applicable for clinical use than conventional PCR, we established a one-step multiplex PCR system for identifying and detecting simultaneously eight medically important Candida species, which uses only one PCR tube per sample. A multiplex PCR method is a rapid tool that allows for the simultaneous amplification of more than one sequence of target DNA in a single reaction, thereby saving time and reagents [32]. The most significant problem with this method is the possibility of hybridization among the different primer sequences. Carvalho et al. previously reported a multiplex PCR strategy allowing the identification of eight Candida species, similar to the present study [33]. This multiplex PCR was based on the amplification of two fragments from ITS1 and ITS2 regions by combining two yeast-specific and eight speciesspecific primers in a single PCR. However, according to our pilot study, because this method was too complicated to distinguish PCR fragment patterns, it was difficult to identify accurately each Candida species. Moreover, it took more than 4 hours to finish the identification.

In the present study, we designed species-specific primers with the already mentioned means, for the identification and detection of eight Candida species using a PCR-based method. These primers were able to distinguish each Candida species and did not display cross-reactivity with representative Candida species other than the eight species targeted in this study. Moreover, we developed a one-step multiplex PCR method with the ability to identify and differentiate eight medically important Candida species (i.e., C. albicans, C. glabrata, C. tropicalis, C. parapsilosis, $C$. dubliniensis, . guilliermondii, C. krusei, and C. lusitaniae) using only one PCR tube per sample.

Our multiplex PCR method is easy because the use of MightyAmp DNA Polymerase Ver.3 (Takara) means that DNA extraction is not necessary, and species identification and detection using this method only takes approximately 2 hours. Thus, the method described herein will allow the prevalence of the eight medically important Candida species and their involvement in various infections to be fully clarified in future studies. 


\section{Author's Contributions}

Fukatsu A, Suzuki H, Asaka K, Ono Y, Fuchigami M, Kobayashi T, Uchibori S, Takahashi Y, Komine C, Konishi Y, Ogura Y, Omori H and Wakami M corrected the data. Fukatsu A, Tsuzukibashi O, Murakami $\mathrm{H}$ and Fukumoto $\mathrm{M}$ drafted and wrote the manuscript. The concept of this manuscript was devised by Fukatsu A. All authors read and approved the final manuscript.

\section{Conflicts of Interest}

The authors declare that there is no conflict of interest.

\section{References}

[1] Wingard, J.R. (1995) Importance of Candida Species Other than C. albicans as Pathogens in Oncology Patients. Clinical Infectious Diseases, 20, 115-125. https://doi.org/10.1093/clinids/20.1.115

[2] Odds, F.C. (1996) Epidemiological Shifts in Opportunistic and Nosocomial Candida Infections: Mycological Aspects. International Journal of Antimicrobial Agents, 6 , 141-144. https://doi.org/10.1016/0924-8579(95)00049-6

[3] Ellis, M.E., Clink, H., Ernst, P., Halim, M.A., Padmos, A., Spence, D., Kalin, M., Hussain, S.M., Qadri, S.M.H., Burnie, J. and Greer, W. (1994) Controlled Study of Fluconazole in the Prevention of Fungal Infections in Neutropenic Patients with Haematological Malignancies and Bone Marrow Transplant Recipients. European Journal of Clinical Microbiology and Infectious Diseases, 13, 3-11. https://doi.org/10.1007/BF02026116

[4] Wingard, J.R., Merz, W.G., Rinaldi, M.G., Miller, C.B., Karp, J.E. and Saral, R. (1993) Association of Torulopsis glabrata Infections with Fluconazole Prophylaxis in Neutropenic Bone Marrow Transplant Patients. Antimicrobial Agents and Chemotherapy, 37, 1847-1849. https://doi.org/10.1128/AAC.37.9.1847

[5] Shepp, D.H., Klosterman, A., Siegel, M.S. and Meyers, J.D. (1985) Comparative Trial of Ketoconazole and Nystatin for Prevention of Fungal Infection in Neutropenic Patients Treated in a Protective Environment. The Journal of Infectious Diseases 152, 1257-1263. https://doi.org/10.1093/infdis/152.6.1257

[6] Fridkin, S.K. and Jarvis, W.R. (1996) Epidemiology of Nosocomial Fungal Infections. Clinical Microbiology Reviews, 9, 499-511. https://doi.org/10.1128/CMR.9.4.499

[7] Tortorano, A.M., Peman, J., Bernhardt, H., Klingspor, L., Kibbler, C.C., Faure, O., Biraghi, E., Canton, E., Zimmermann, K., Seaton, S., Grillot, R. and ECMM Working Group on Candidaemia (2004) Epidemiology of Candidaemia in Europe: Results of 28-Month European Confederation of Medical Mycology (ECMM) Hospital-Based Surveillance Study. European Journal of Clinical Microbiology and Infectious Diseases, 23, 317-322. https://doi.org/10.1007/s10096-004-1103-y

[8] Wenzel, R.P. and Edmond, M.B. (2001) The Impact of Hospital-Acquired Bloodstream Infections. Emerging Infectious Diseases, 7, 174-177.

[9] Diekema, D.J., Messer, S.A., Brueggemann, A.B., Coffman, S.L., Doern, G.V., Herwaldt, L.A. and Pfaller, M.A. (2002) Epidemiology of Candidemia: 3-Year Results from the Emerging Infections and the Epidemiology of Iowa Organisms Study. Journal of Clinical Microbiology, 40, 1298-1302. https://doi.org/10.1128/JCM.40.4.1298-1302.2002 
[10] Pfaller, M.A. and Diekema, D.J. (2002) Role of Sentinel Surveillance of Candidemia: Trends in Species Distribution and Antifungal Susceptibility. Journal of Clinical Microbiology, 40, 3551-3557. https://doi.org/10.1128/JCM.40.10.3551-3557.2002

[11] Kao, A.S., Brandt, M.E., Pruitt, W.R., Conn, L.A., Perkins, B.A., Stephens, D.S. Baughman, W.S., Reingold, A.L., Rothrock, G.A., Pfaller, M.A., Pinner, R.W. and Hajjeh, R.A.(1999) The Epidemiology of Candidemia in Two United States Cities: Results of a Populationbased Active Surveillance. Clinical Infectious Diseases, 29, 1164-1170. https://doi.org/10.1086/313450

[12] Berenguer, J., Buck, M., Witebsky, F., Stock, F., Pizzo, P.A. and Walsh, T.J. (1993) Lysis-Centrifugation Blood Cultures in the Detection of Tissue-Proven Invasive Candidiasis. Disseminated versus Single-Organ Infection. Diagnostic Microbiology and Infectious Disease, 17, 103-109. https://doi.org/10.1016/0732-8893(93)90020-8

[13] Fenn, J.P., Segal, H., Barland, B., Denton, D., Whisenant, J., Chun, H., Christofferson, K., Hamilton, L. and Carroll, K. (1994) Comparison of Updated Vitek Yeast Biochemical Card and API 20C Yeast Identification Systems. Journal of Clinical Microbiology, 32, 1184-1187. https://doi.org/10.1128/JCM.32.5.1184-1187.1994

[14] Buchman, T.G., Rossier, M., Merz, W.G. and Charache, P. (1990) Detection of Surgical Pathogens by in Vitro DNA Amplification. Part I. Rapid Identification of Candida albicans by in Vitro Amplification of a Fungus-Specific Gene. Surgery, 108, 338-346.

[15] Burgener-Kairuz, P., Zuber, J.P. and Jaunin, P. (1994) Rapid Detection and Identification of Candida albicans and Torulopsis (Candida) glabrata in Clinical Specimens by Species-Specific Nested PCR Amplification of a Cytochrome P-450 Lanosterol-Alpha-Demethylase (L1A1) Gene Fragment. Journal of Clinical Microbiology, 32, 1902-1907. https://doi.org/10.1128/JCM.32.8.1902-1907.1994

[16] Crampin, A.C. and Matthews, R.C. (1993) Application of the Polymerase Chain Reaction to the Diagnosis of Candidosis by Amplification of an HSP 90 Gene Fragment. Journal of Medical Microbiology, 39. 233-238. https://doi.org/10.1099/00222615-39-3-233

[17] Fujita, S., Lasker, B.A., Lott, T.J., Reiss, E. and Morrison, C.J. (1995) Microtitration Plate Enzyme Immunoassay to Detect PCR-Amplified DNA from Candida Species in Blood. Journal of Clinical Microbiology, 33, 962-967. https://doi.org/10.1128/JCM.33.4.962-967.1995

[18] Miyakawa, Y., Mabuchi, T., Kagaya, K. and Fukazawa, Y. (1992) Isolation and Characterization of a Species-Specific DNA Fragment for Detection of Candida albicans by Polymerase Chain Reaction. Journal of Clinical Microbiology, 30, 894-900. https://doi.org/10.1128/JCM.30.4.894-900.1992

[19] Chang, H.C., Leaw, S.N., Huang, A.H., Wu, T.L. and Chang, T.C. (2001) Rapid Identification of Yeasts in Positive Blood Cultures by a Multiplex PCR Method. Journal of Clinical Microbiology, 39, 3466-3471. https://doi.org/10.1128/JCM.39.10.3466-3471.2001

[20] Elie, C.M., Lott, T.J., Reiss, E. and Morrison, C.J. (1998) Rapid Identification of Candida Species with Species-Specific DNA Probes. Journal of Clinical Microbiology, 36, 3260-3265. https://doi.org/10.1128/JCM.36.11.3260-3265.1998

[21] Williams, D.W., Wilson, M.J., Lewis, M.A. and Potts, A.J. (1995) Identification of Candida Species by PCR and Restriction Fragment Length Polymorphism Analysis of Intergenic Spacer Regions of Ribosomal DNA. Journal of Clinical Microbiology, 33, 2476-2479. https://doi.org/10.1128/JCM.33.9.2476-2479.1995

[22] Tsuzukibashi, O., Uchibori, S., Kobayashi, T., Saito, M., Umezawa, K., Ohta, M. and 
Shinozaki, K.N. (2015) A Selective Medium for the Isolation of Microbacterium Species in Oral Cavities. Journal of Microbiological Methods, 116, 60-65. https://doi.org/10.1016/j.mimet.2015.06.016

[23] Crist Jr., A.E., Johnson, L.M. and Burke, P.J. (1996) Evaluation of the Microbial Identification System for Identification of Clinically Isolated Yeasts. Journal of Clinical Microbiology, 34, 2408-2410. https://doi.org/10.1128/JCM.34.10.2408-2410.1996

[24] Latouche, G.N., Daniel, H.M., Lee, O.C., Mitchell, T.G., Sorrell, T.C. and Meyer, W. (1997) Comparison of Use of Phenotypic and Genotypic Characteristics for Identification of Species of the Anamorph Genus Candida and Related Teleomorph Yeast Species. Journal of Clinical Microbiology, 35, 3171-3180.

https://doi.org/10.1128/JCM.35.12.3171-3180.1997

[25] Heelan, J.S., Sotomayor, E., Coon, K. and D’Arezzo, J.B. (1998) Comparison of the Rapid Yeast Plus Panel with the API20C Yeast System for Identification of Clinically Significant Isolates of Candida Species. Journal of Clinical Microbiology, 36, 1443-1445. https://doi.org/10.1128/JCM.36.5.1443-1445.1998

[26] Coleman, D.C., Sullivan, D.J., Bennett, D.E., Moran, G.P., Barry, H.J. and Shanley, D.B. (1997) Candidiasis: The Emergence of a Novel Species, Candida dubliniensis. AIDS, 11, 557-567. https://doi.org/10.1097/00002030-199705000-00002

[27] Sullivan, D.J., Moran, G., Donnelly, S., Pinjon, E., McCartan, B., Shanley, D.B. and Coleman, D.C. (1999) Candida dubliniensis. An Update. Revista Iberoamericana de Micología, 16, 72-76.

[28] Gutierrez, J., Morales, P., Gonzalez, M.A. and Quindos, G. (2002) Candida dubliniensis, a New Fungal Pathogen. Journal of Basic Microbiology, 42, 207-227. https://doi.org/10.1002/1521-4028(200206)42:3<207::AID-JOBM207>3.0.CO;2-C

[29] Bassetti, M., Righi, E., Costa, A., Fasce, R., Molinari, M.P., Rosso, R., Pallavicini, F.B. and Viscoli, C. (2006) Epidemiological Trends in Nosocomial Candidemia in Intensive Care. BMC Infectious Diseases, 6, Article No. 21. https://doi.org/10.1186/1471-2334-6-21

[30] Pfaller, M.A., Messer, S.A., Hollis, R.J., Jones, R.N., Doern, G.V., Brandt, M.E., Hajjeh, R.A. (1999) Trends in Species Distribution and Susceptibility to Fluconazole among Blood Stream Isolates of Candida Species in the United States. Diagnostic Microbiology and Infectious Disease, 33, 217-222.

https://doi.org/10.1016/S0732-8893(98)00160-6

[31] Moran, G.P., Sullivan, D.J., Henman, M.C., McCreary, C.E., Harrington, B.J., Shanley, D.B. and Coleman, D.C. (1997) Antifungal Drug Susceptibilities of Oral Candida dubliniensis Isolates from Human Immunodeficiency Virus (HIV)-Infected and Non-HIV-Infected Subjects and Generation of Stable Fluconazoleresistant Derivatives in Vitro. Antimicrobial Agents and Chemotherapy, 41, 617-623.

https://doi.org/10.1128/AAC.41.3.617

[32] Henegariu, O., Heerema, N.A., Dlouhy, S.R., Vance, G.H. and Vogt, P.H. (1997) Multiplex PCR: Critical Parameters and Step-by-Step Protocol. BioTechniques, 23, 504-511. https://doi.org/10.2144/97233rr01

[33] Carvalho, A., Costa-de-Oliveira, S., Martins, M.L., Pina-Vaz, C., Rodrigues, G., Ludovico, P. and Rodrigues, F. (2007) Multiplex PCR Identification of Eight Clinically Relevant Candida Species. Medical Mycology, 45, 619-627. https://doi.org/10.1080/13693780701501787 